\title{
Multidimensional imaging of the partonic structure of hadrons with an Electron-Ion Collider
}

\author{
Salvatore Fazio* \\ Brookhaven National Laboratory, Upton, New York, U.S.A. 11967 \\ E-mail: sfaziodonl.gov
}

The American 2015 nuclear physics long-range plan and the 2018 report from the American National Academy of Sciences endorsed the realization of an electron-ion collider (EIC) as top priority for a next large construction project in the United States. With the design of an EIC, advancements in theory and further development of phenomenological tools, we are now preparing for the next step in subnuclear tomographic imaging. The collider's large range of center-ofmass energies in combination with very high luminosity and polarization of both the lepton and the hadron beams, will open a unique opportunity for very high precision measurements of both cross sections and spin-asymmetries. This will allow us for a detailed investigation of the partonic substructure of hadrons in multi-dimensions, as well as addressing the role of orbital angular momentum with respect to the nucleon spin. Generalized parton distributions (GPDs) describe the multi-dimensional partonic structure of a nucleon in coordinate space, providing new information about the internal dynamics of quarks and gluons. The extraction of GPDs by measuring hard exclusive processes in electron+proton and electron+ion collisions, with all related probes, is an essential element of the EIC science program. We highlight key measurements, experimental challenges, and finally discuss the EIC's expected impact over the current knowledge of the partonic 3D-structure of hadrons.

23rd International Spin Physics Symposium - SPIN2018 -

10-14 September, 2018

Ferrara, Italy

\footnotetext{
* Speaker.
} 


\section{The electron-ion collider}

The EIC project [1] is a major new research facility to advance the long-term vision for $\mathrm{Nu}$ clear Physics to discover and understand the emergent phenomena of QCD, e.g. the creation of mass and spin of the visible matter. In the 2015 Long Range Planning for Nuclear Physics in the United States (U.S.) [2], the American National Science Advisory Committee recommended the realization of a high-energy high-luminosity polarized EIC as "the highest priority for new facility construction following the completion of FRIB". In July 2018, the U.S. National Academy of Sciences endorsed this project as "a unique facility in the world that would answer science questions that are compelling, fundamental, and timely" [3].

Currently there are two proposals to construct an EIC in the United States. One option would involve the addition of a hadron accelerator complex to the existing CEBAF facility at the Thomas Jefferson National Accelerator Facility (JLAB), the so-called JLEIC project. The other option would be to add an electron accelerator to the existing Relativistic Heavy-Ion Collider (RHIC) facility at Brookhaven National Laboratory (BNL), a project know as eRHIC. Despite the two competing proposals and strategies for an EIC, the overriding goal is the same: to build an accelerator, which is flexible in terms of ion species (proton to uranium) and beam energy. Both proposals plan for a final per-nucleon centre-of-mass energies ranging from $20 \mathrm{GeV}$ to $100 \mathrm{GeV}$ for polarized lepton+proton collisions, upgradable up to $140 \mathrm{GeV}$. With a peak luminosity close to $10^{34} \mathrm{~cm}^{-2} \mathrm{~s}^{-1}$, an EIC will exceed of three orders of magnitude what was achieved at HERA.

\section{Tomographic images of quarks and gluons in coordinate space}

With its wide range in energy, nuclear beams and high luminosity, EIC will offer an unprecedented opportunity for precision measurements, allowing us to study the momentum and spacetime distribution of gluons and sea quarks in nucleons and nuclei. One of the main goals will be a precise determination of the Generalized Parton Distribution functions (GPDs). These are a generalization of the one dimensional Parton Distribution Functions (PDFs) and can describe the correlations between the longitudinal momentum of quarks and gluons and their position in the transverse spatial plane in a nucleon. Moreover, one set of GPDs is sensitive to the contribution of the orbital motion of quarks and gluons to the nucleon spin.

GPDs can be accessed through measuring exclusive processes in electron+nucleon scattering. The important observables sensitive to the GPDs are the differential cross section as a function of the four-momentum transfer at the proton vertex, $|t|$, and the beam-helicity asymmetries.

A golden measurement toward the determination of the whole set of GPDs is Deeply Virtual Compton Scattering (DVCS), which is the exclusive production of a real photon. This theoretically and experimentally clean process is sensitive to both quarks and gluons (via the evolution of the virtuality of the exchanged photon, $Q^{2}$ ). For the purpose of the cross section measurement it is important to remove from the signal the background coming from the Bethe-Heithler $(\mathrm{BH})$ events. The latter is essentially a QED process, known to an uncertainty in the order of $\sim 3-4 \%$ coming from the uncertainty on the proton form factor, with the same final state topology as DVCS and can be subtracted from the signal using a Monte Carlo (MC) technique. 


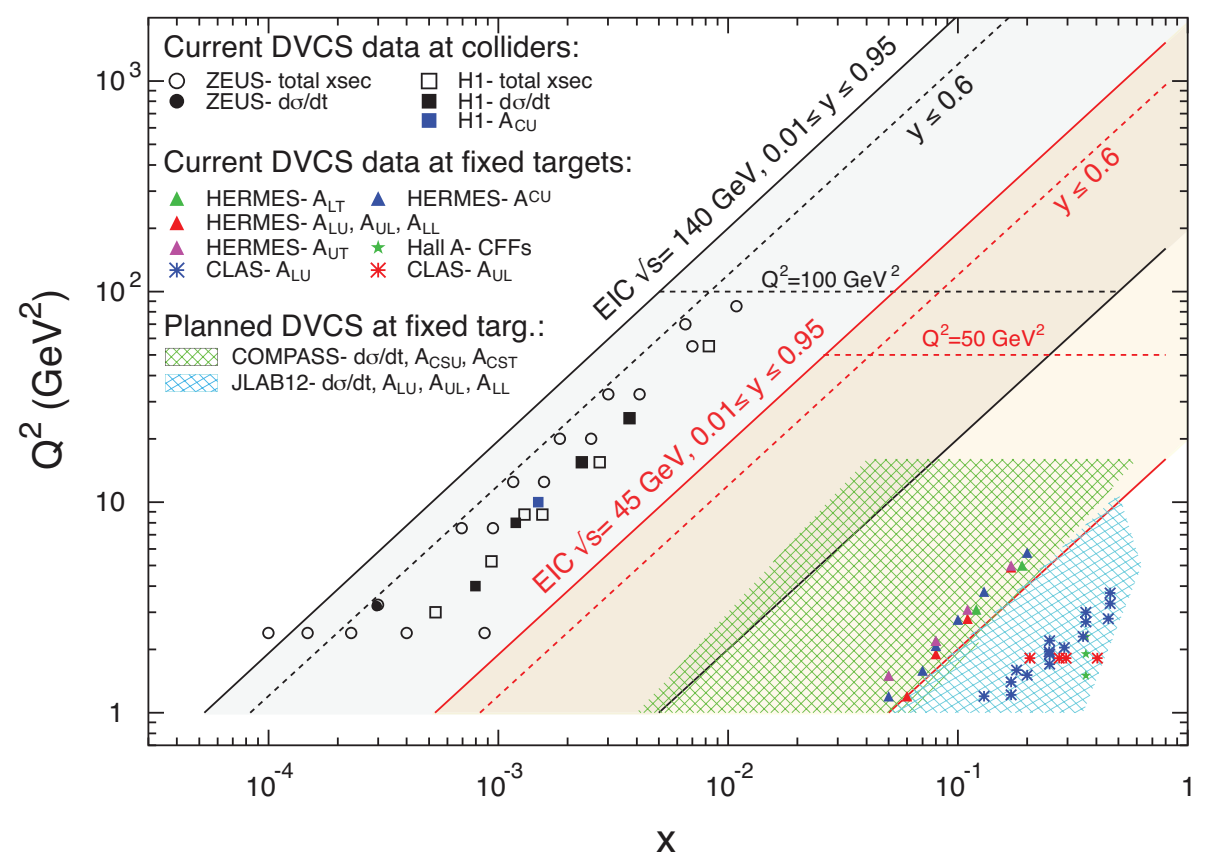

Figure 1: The EIC phase space for $\sqrt{s}=45 \mathrm{GeV}$ (red) and $\sqrt{s}=140 \mathrm{GeV}$ (black) compared with available DVCS data and expected future DVCS experiments.

Presently available DVCS measurements provide some limited information on GPDs and more precise data, in a wider phase space and including transversely polarized target spin asymmetry, are required to pin them down [4]. The EIC will cover a much larger phase-space, see Figure 1, connecting the domain typical of fixed target experiments at large $x$ whit that of collider experiments at small $x$, and will help to quantify QCD phenomena.

Samples of pseudo-data have been generated with MILOU [5] - which simulates both the DVCS and the $\mathrm{BH}$ processes together with their interference term. The $\mathrm{BH}$ contamination has been investigated for each $Q^{2}, x_{B j},|t|$ bin as a function of the inelasticity, $y$. After all BH suppression criteria have been applied it was found that for higher EIC c.o.m. energies the BH contamination grows from negligible (at low- $y$ ) to about $70 \%$ at $y \sim 0.6$, whereas for lower energies it grows faster and can be dominant at large- $y$, although most of the statistics is contained in the safe region of $y<0.3$. These Simulated events, corresponding to a collected luminosity of $10 \mathrm{fb}^{-1}$, have been used to measure the $|t|$-differential cross section and the spin-asymmetries. All the variables have been smeared according to the expected experimental resolution. A logarithmic binning of $x$ and $Q^{2}$ has been applied as in Figure 2, and $|t|$ has been binned three times larger than the expected resolution.

An access to GPDs requires a large data set with small errors. As an example of the precision achievable at the EIC, Figure 2 shows the expected uncertainty for a measurement of the DVCS $|t|$-differential cross section in a particular $x, Q^{2}$ bin. Figure 3 shows the expected uncertainty for the transverse target-spin asymmetry $\left(A_{U T}\right)$ as a function of the azimuthal angle $\phi$ between the production and the scattering planes for a particular $x, Q^{2},|t|$ bin, compared to theoretical expectations. The simulation proves that an EIC can perform accurate measurements of cross sections and asym- 

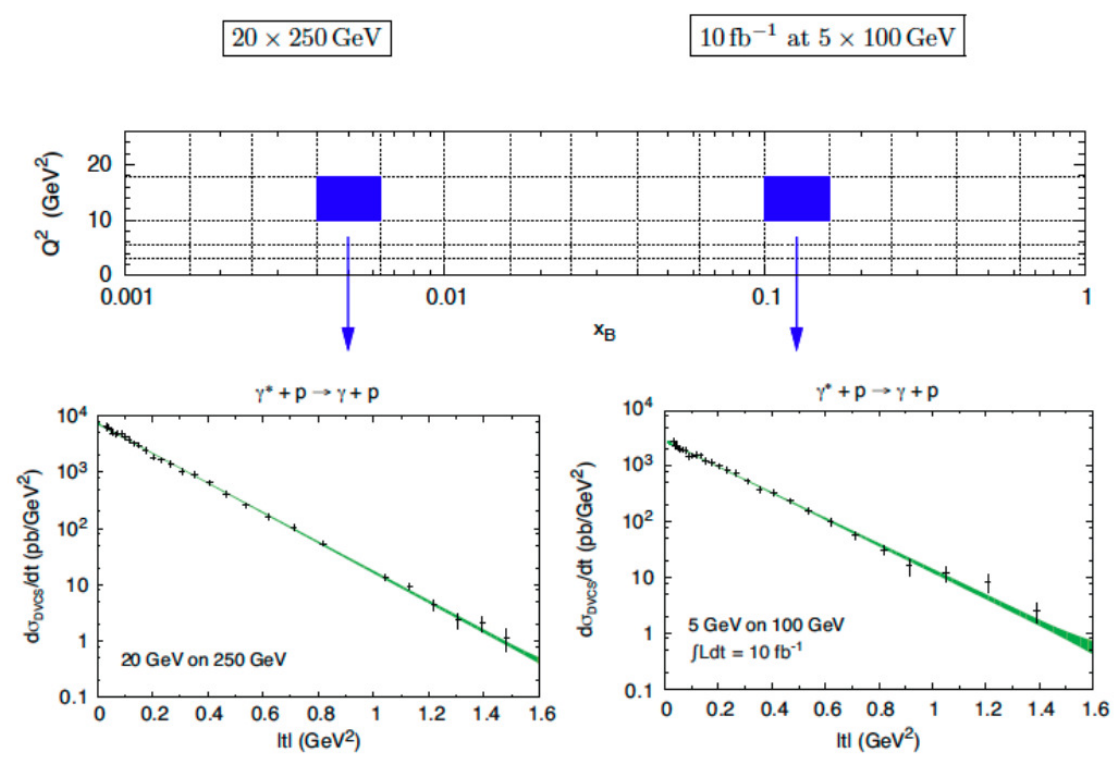

Figure 2: Expected uncertainties for a DVCS $|t|$-differential cross section measurement in a particular $x, Q^{2}$ bin at a higher (left) and a lower (right) beam-energy configuration of an EIC.
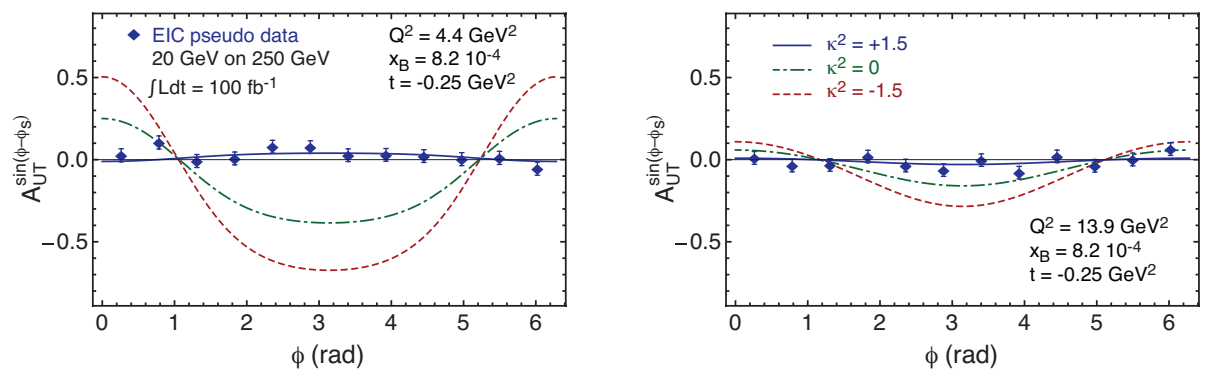

Figure 3: Expected uncertainties for a DVCS $A_{U T}$ compared to theory model with large positive (solid), vanishing (dot-dashed), and large negative (dashed) $E^{\text {sea }}$ contributions.

metries in a very fine binning and with a very low statistical uncertainty. Measurements at EIC are often limited by systematics. For the purposes of the present study, a systematic uncertainty of 5\% has been assumed, based on the experience achieved at HERA and the expected acceptance and technology improvements of future EIC detectors. The overall systematic uncertainty due to the uncertainty on the measurement of luminosity is not shown on the plots, since it simply affects the normalization of the cross section measurement.

A global fit, including the EIC simulated data together with all the data presently available has been done. Figure 4 shows how an EIC can largely improve the knowledge on GPD H for gluons. Moreover, a precise measurement of the transversely polarized target spin asymmetry $A_{U T}$, which allows for a decomposition of GPD $\mathrm{H}$ and $\mathrm{E}$ contributions, leads to the accurate extraction of GPD E, which at the moment remains almost unconstrained [4], providing an estimate of the angular momentum carried by sea quarks.

Fourier-transforming the GPDs, it is possible to obtain the quarks and gluons distributions in 

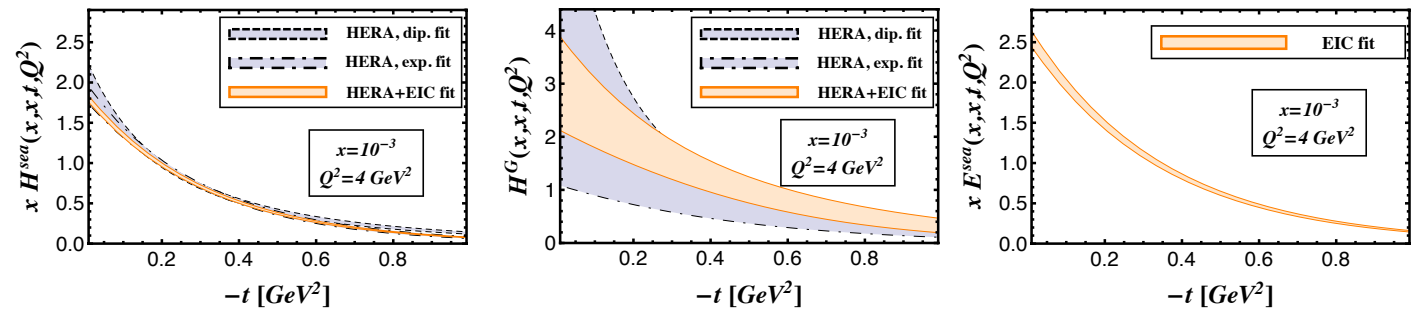

Figure 4: Extraction of GPD H for sea quarks (top) and gluons (center) and GPD E for sea quarks (bottom) in a particular $x, Q^{2}$ bin. The violet band is the uncertainty obtained excluding the EIC pseudo-data from the global fit procedure.

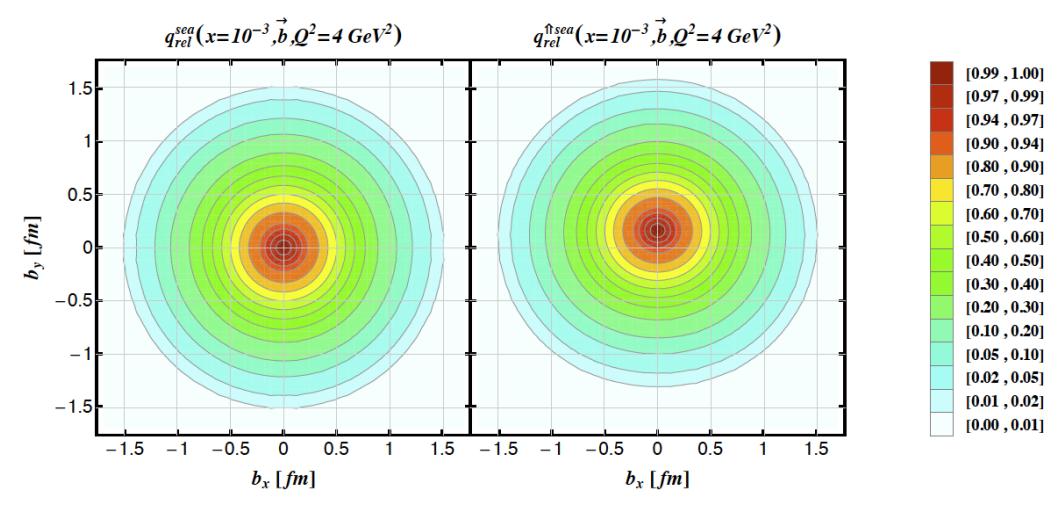

Figure 5: Tomographical picture of the sea-quarks distribution in the impact parameter space for an unpolarized (left) and a polarized (right) proton beam.

the impact parameter space. Figure 5 shows an example of a tomographic $(2+1 \mathrm{D})$ picture of the see-quarks distribution as resulting from the EIC pseudo-data analysis, in a particular bin, for the case of an unpolarized and a polarized proton-beam. The shift observed in the polarized case comes from the GPD E contribution.

DVCS measurements generally involve all the spin-flavor components of the quark GPDs and rely on polarization observables and proton/neutron comparison to separate them; gluons are accessible only through QCD evolution. Deeply virtual meson production (DVMP) can directly probe specific components of the GPDs, complementing the DVCS measurements [6]. This includes (a) probing gluon GPDs in heavy meson electroproduction $(J / \psi, \phi)$; (b) separating quark flavor GPDs with light vector meson production $\left(\rho^{0}, \rho^{+}, \omega\right)$; (c) accessing quark transversity with light pseudoscalar mesons $\left(\pi^{0}, \eta\right)$ and two-meson final states; (d) probing strangeness in the nucleon with strange meson electroproduction $\left(K, K^{*}\right)$.

While current impact studies are solely based on DVCS, an EIC can provide high precision measurements of DVMP processes. A simulation of an EIC measurement of the $|t|$-differential cross section for the $j / \psi$ meson production citeAccardi:2012qut for a particular bin of $x_{B j}$ and $Q^{2}+$ $M_{j / \psi}^{2}$, at the center-of-mass (c.o.m.) energy of $\sqrt{s}=140 \mathrm{GeV}$, assuming a collected luminosity of $10 \mathrm{fb}^{-1}$, is shown in Figure 6 (left). The same figure, on the right-end side, shows an EIC simulation of $Y$ production [7] at higher $x_{B j}, Q^{2}+M_{j / \psi}^{2}$ and for a lower c.o.m.: $\sqrt{s}=63 \mathrm{GeV}$, assuming a collected luminosity of $100 \mathrm{fb}^{-1}$. 

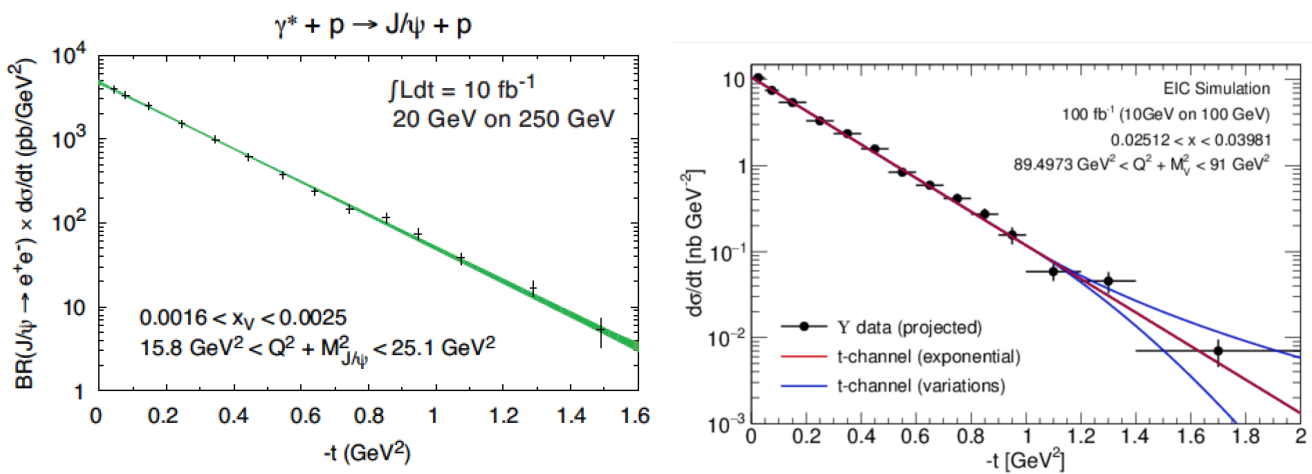

Figure 6: Expected experimental accuracy for a $|t|$-differential cross section of $j / \psi$ (left) and $Y$ (right) meson production processes.

A Fourier-transform of the $|t|$-differential cross section for the production of heavy vector mesons is already a good estimate of the uncertainty achievable on the gluon distributions in the impact parameter space, though it still contains a contribution from the small but finite size of the meson, which needs to be disentangled in a full GPD analysis. Measuring the transverse proton spin asymmetry for heavy meson production will, in addition, give constraints on the GPD E for gluons and thus strongly complement what can be achieved with DVCS.
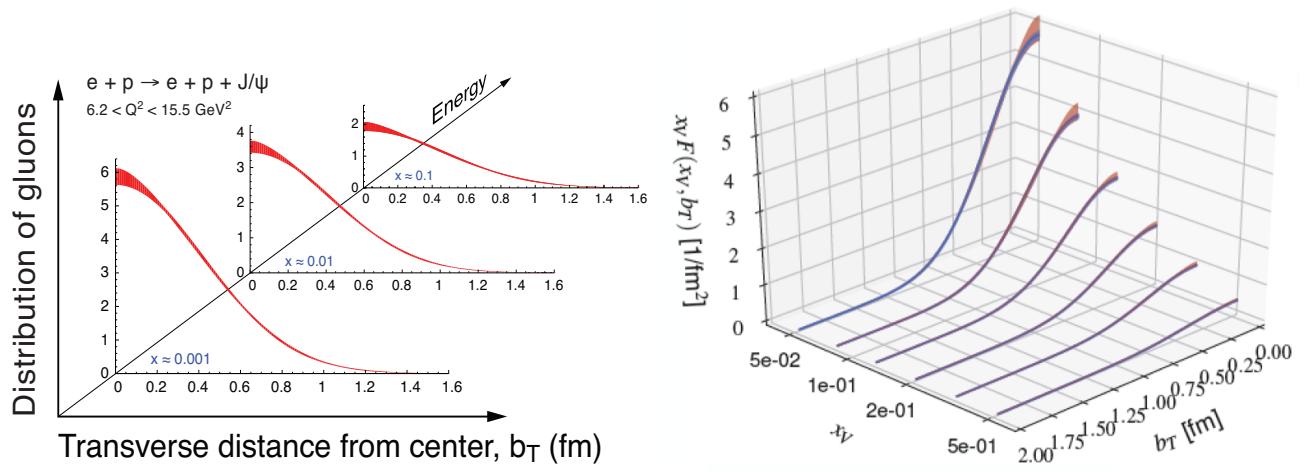

Figure 7: Projected EIC uncertainties on the measurement of the gluon distribution versus the transverse distance from the center of the nucleon, resulting from differential cross section measurements of $j / \psi$ (left) and $Y$ (right). The gluon distributions uncertainty bands are plotted for a fixed $Q^{2}$ bin and three different values of Bjorken's $x$.

Figure 7 shows the projected gluon distributions measurable at the EIC in a particular bin of $Q^{2}$ and at different values of $x$. We see from the Figure that the data will enable us to accurately probe the spatial distribution of gluons over two orders of magnitude in $x$, up to the region where the dominant partons are valence quarks.

Furthermore, a unique opportunity offered by an EIC is to go beyond the imaging of a single proton and study the multidimensional distribution of quarks and gluons in nuclei. These studies will lead to quantify the effects of a nuclear environment on the PDFs and the GPDs. The precision achievable at an EIC in measuring exclusive processes in electron+nucleus scattering has been explored using the purposely developed Sartre [8] event generator specialized for diffractive 
exclusive vector meson production and based on the saturation-regime (bSat) dipole model [9] and its linearization, the non-saturation (bNonSat) model [10]. The parameters of both models were tuned to describe the $e+p$ HERA data. The produced events were passed through an experimental filter and scaled to reflect an integrated luminosity of $10 \mathrm{fb}^{-1} / \mathrm{A}$. A conservative $|t|$-resolution of $5 \%$ has been assumed.
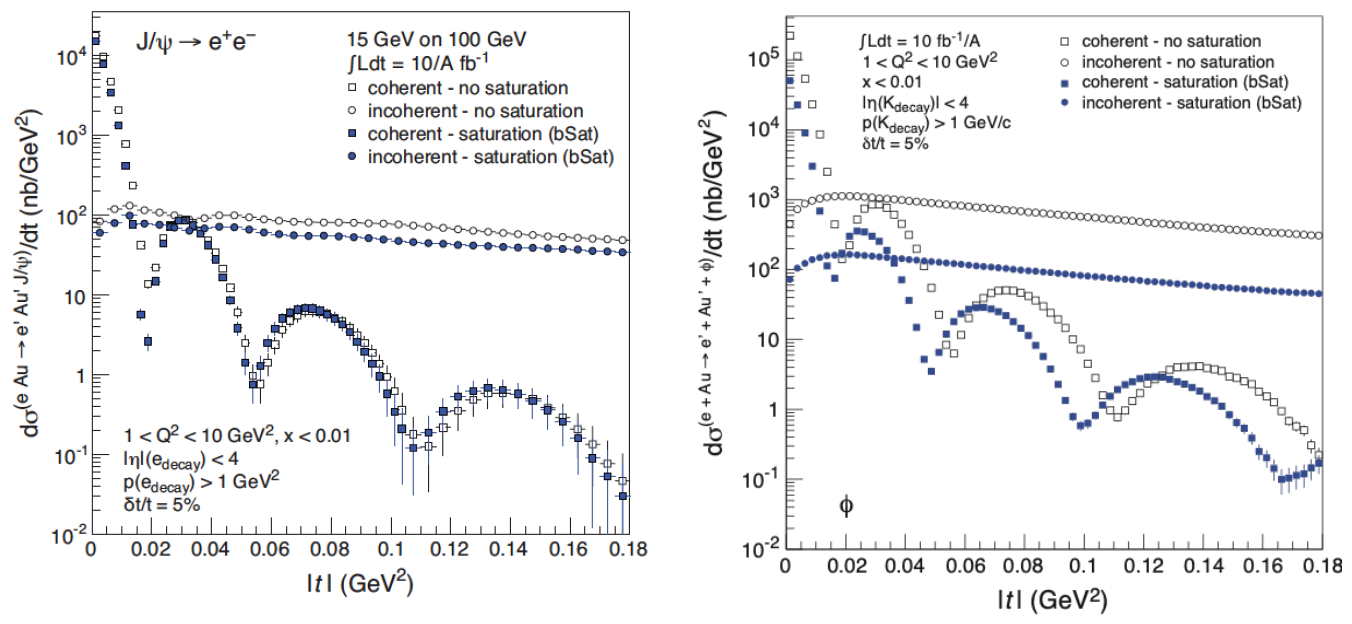

Figure 8: $|t|$-differential cross section distributions for exclusive $j / \psi$ (left) and $\phi$ (right) production in coherent (blue circles) and incoherent (open circles) events in diffractive $e+$ Au collisions at an EIC.

Figure 8 shows the $|t|$-differential cross section for the production of $j / \psi$ (left) and $\phi$ (right) mesons in $e+\mathrm{Au}$ collisions. The cross section measurement has been simulated separately for saturation and non-saturation models. Figure 8 compares the values of the coherent cross sections and the incoherent one, a process where the the nucleus brakes up after the collision. Generally, only the sum of the coherent and incoherent parts of the cross section is measured. Therefore it is crucial to properly design the detector in order to experimentally disentangle the two contributions unambiguously. This might be achieved through the detection of emitted neutrons (e.g. by zerodegree calorimeters) from the nuclear breakup and, optionally, the breakup products in detectors along the beam-line (Roman-Pots) in the incoherent case. Similarly to the case of a single proton, the Fourier transform of the coherent cross section distributions in Figure 8 can be used to obtain information about the gluon distribution in impact parameter space.

\section{References}

[1] A. Accardi, A., J. L. Albacete, M. Anselmino et al., Eur. Phys. J. A (2016) 52: 268.

[2] https://science.energy.gov/np/nsac/

[3] NAS report on EIC physics

[4] E. C. Aschenauer, S. Fazio, K. Kumericki, and D. Mueller, JHEP 09 (2013) 093.

[5] E. Perez, L. Schoeffel, and L. Favart, arXiv:hep-ph/0411389.

[6] M. Diehl, Phys. Rept. 388 (2003) 41-277. 
[7] S. Joosten, arXiv: 1803.08615

[8] T. Toll and T. Ullrich, Comput. Phys. Commun. 185 (2014) 1835-1853.

[9] H. Kowalski, L. Motyka, and G. Watt, Phys. Rev. D 74, 074016 (2006).

[10] H. Kowalski and D. Teaney, Phys. Rev. D 68, 114005 (2003). 